\title{
EWSR1/FLI1 Fusion Protein Type 2
}

National Cancer Institute

\section{Source}

National Cancer Institute. EWSR1/FLI1 Fusion Protein Type 2. NCI Thesaurus. Code C99207.

A fusion protein ( $526 \mathrm{aa}, \sim 57 \mathrm{kDa}$ ) encoded by the EWSR1/FLI1 fusion gene type 2 . This protein is comprised of the transactivation domain of the RNA-binding protein EWS fused to the C-terminal domain of the Friend leukemia integration 1 transcription factor protein, which includes the ETS DNA binding domain. 